\title{
Analyse et traitement par plasma froid des eaux de rejet de « l'abattoir des Brasseries » à Yaoundé
}

\author{
Jourdin GONGWALA $^{1 *}$, ABBA PALTAHE ${ }^{1}$, Gaston PAYOM ${ }^{2}$ et Daniel NJOPWOUO ${ }^{2}$ \\ ${ }^{1}$ Université de Maroua, ENS B.P.55 Maroua, Cameroun. \\ ${ }^{2}$ Université de Yaoundé I, Faculté des Sciences B.P. 812 Yaoundé, Cameroun. \\ *Auteur correspondant, E-mail : gongwala@yahoo.fr ; Tél. (00237)75180348
}

\section{RESUME}

Des échantillons des eaux usées de «l'abattoir des Brasseries » à Yaoundé, prélevés ont été analysés et traités par plasma froid. Les résultats d'analyse révèlent que lesdites eaux sont basiques $(\mathrm{pH}=8.65)$, très conductrices (conductivité électrique $\left.=1625 \mu \mathrm{Scm}^{-1}\right)$, fortement polluées $\left(\mathrm{DBO}_{5}=1000 \mathrm{mgL}^{-1} \mathrm{~d}^{\prime} \mathrm{O}_{2}, \mathrm{DCO}=\right.$ $2390 \mathrm{mgL}^{-1} \mathrm{~d}^{\prime} \mathrm{O}_{2}$ et $200 \mathrm{mgL}^{-1}$ d'ammonium) et biodégradables $\left(\mathrm{DBO}_{5} / \mathrm{DCO}=0.42\right)$. La teneur élevée en chlorures $\left(1347 \mathrm{mgL}^{-1}\right)$ et l'absence des métaux lourds ont été notées. Après traitement par plasma, on observe une réduction de $50 \%$ de la $\mathrm{DBO}_{5}$ en 10 minutes, le débit du gaz plasmagène étant fixé à $1000 \mathrm{Lh}^{-1}$. L'efficacité du plasma froid à décharge électrique rampante (glidarc) a été une fois de plus prouvée pour la réduction de la pollution environnementale.

(c) 2012 International Formulae Group. All rights reserved.

Mots clés: Eaux usées, biodégradables, glidarc, pollution.

\section{INTRODUCTION}

Les industries africaines en général et camerounaises en particulier sont de plus en plus confrontées aux problèmes de protection de l'environnement. En effet, la croissance démographique, l'urbanisation rapide, le changement de mode de vie ainsi que le développement économique pèsent lourdement sur les ressources hydriques dans le monde entier en les polluants (Rodier, 2009). Les abattoirs figurent parmi les structures polluantes (Labioui et al., 2007 ; Gnokam Zumgang, 2008). Les eaux rejetées sont de façon générale polluées par des matières d'origine agricole, chimique, industrielle et domestique. Ces polluants ont des effets néfastes et variés sur l'environnement : maladies hydriques, eutrophisation des corps d'eau, etc (Rodier, 2009). La faune et la flore, ainsi que l'homme sont de ce fait en danger. Ce dernier doit donc entreprendre des actions concrètes en vue de la prévention, de la réduction et même de l'éradication de la pollution (Doubla, 2002). Pour ce faire, il faut mettre en œuvre des systèmes d'épuration et de traitement des eaux usées avant leur rejet dans le milieu récepteur. Notre objectif dans ce travail était de dépolluer les eaux usées de l'abattoir par le procédé de plasma d'air humide. En effet, la technique de traitement par plasma offre de nos jours plus de commodité et d'avantages par rapport aux méthodes classiques (dégrillage, dessablage, dégraissage- 
déshuilage, tamisage, décantation, filtration sur membrane, dégazage, floculation, coagulation, flottation, oxydation, réduction, extraction liquide-liquide, précipitation, épuration biologique par boue activée, épuration par digestion anaérobique, épandage, lagunage, etc) somme toute complémentaires (Brisset, 2009; Rodier, 2009).

\section{MATERIEL ET METHODES}

Le $\mathrm{pH}$ a été mesuré par un $\mathrm{pH}$-mètre modèle SCHOTT GERATE type pH-meter CG 818. La température et la conductivité électrique ont été obtenues grâce à un conductimètre modèle $\mathrm{HACH} \mathrm{CO} 150$ type conductivity meter auquel est incorporé le thermomètre. La détermination des matières en suspension dans l'eau s'est fait par filtration simple. Les dosages volumétrique, spectrophotométrique ou d'absorption atomique ont permis d'évaluer les autres paramètres (Rodier, 2009).

Le traitement a été effectué par plasma froid à décharge électrique. Le plasma se définit comme un mélange gazeux globalement neutre du point de vue électrique contenant des particules chargées (ions et électrons), des particules neutres (molécules, atomes et radicaux), le plus souvent dans des états excités (Fridman, 2008 ; Held, 1994). Le plasma est une technique de pointe adaptée à la dépollution avec des avantages nombreux : absence de catalyseurs, coût réduit, efficacité de réduction en peu de temps, etc (Brisset, 2009).

Dans le cadre de ce travail, le plasma froid obtenu par décharge électrique glissante appelé glidarc a été utilisé (Doubla, 2002). Son principe repose sur l'établissement d'une décharge électrique entre deux conducteurs divergents portés à une différence de potentiel convenable; la décharge est soufflée par un jet gazeux dirigé selon l'axe du réacteur. La décharge éclate en un panache non thermique qui lèche une cible et ses espèces réagissent à l'interface cible-plasma comme indiqué à la Figure 1 (Doubla, 2002 ; Brisset, 2009).

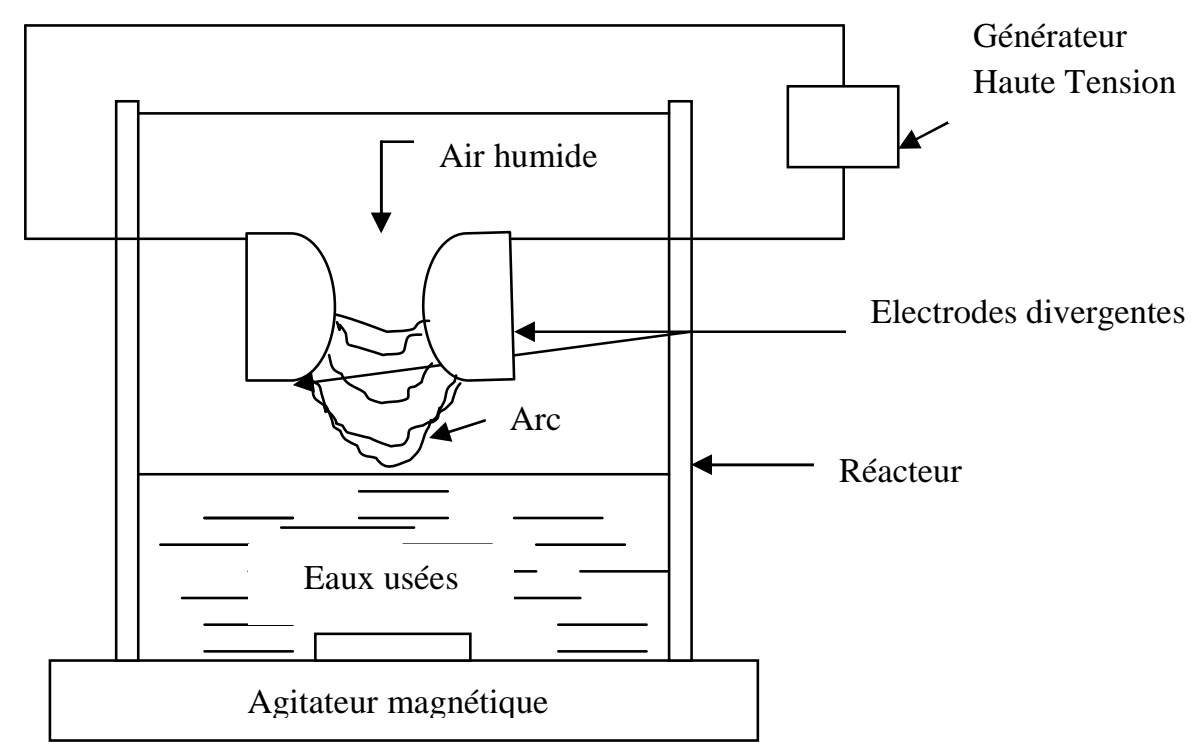

Figure 1: Dispositif expérimental du glidarc. 


\section{RESULTATS}

Le technique plasma froid de type glidarc a permis d'obtenir les résultats contenus dans les Tableaux 1 et 2 . Les paramètres suivis sont le $\mathrm{pH}$, la température, la conductivité électrique, les matières en suspension (MES), la demande chimique en oxygène (DCO), la demande biochimique en oxygène en cinq jours $\left(\mathrm{DBO}_{5}\right)$.

Des courbes d'évolution de ces paramètres en fonction du temps et du débit du gaz plasmagène ont été représentées pour faciliter l'interprétation des résultats (Figures 2 à 13).

A ces résultats s'ajoutent en complément des paramètres mesurés à partir de l'échantillon brut pouvant expliquer la teneur ou l'évolution des paramètres suivis ou renseigner davantage sur l'état de pollution des eaux de cet abattoir (Tableau 3).

Tableau 1: Influence du temps de traitement (à $\left.1000 \mathrm{Lh}^{-1}\right)$.

\begin{tabular}{lcccccc}
\hline Temps (min) & $\mathbf{0}$ & $\mathbf{1}$ & $\mathbf{2}$ & $\mathbf{5}$ & $\mathbf{1 0}$ & $\mathbf{1 5}$ \\
\hline $\mathrm{T}\left({ }^{\circ} \mathrm{C}\right)$ & 28.2 & 28.2 & 27.7 & 28.5 & 30 & 30 \\
$\mathrm{pH}$ & 8.65 & 8.77 & 8.86 & 9.04 & 9.11 & 9.16 \\
Conductivité $\left(\mu \mathrm{Scm}^{-1}\right)$ & 1625 & 1756 & 1796 & 1726 & 1716 & 1875 \\
$\mathrm{MES}\left(\mathrm{mgL}^{-1}\right)$ & 775 & 750 & 850 & 675 & 775 & 900 \\
$\mathrm{DCO}\left(\mathrm{mgL}^{-1} \mathrm{~d}^{\prime} \mathrm{O}_{2}\right)$ & 2390 & 2450 & 2120 & 2340 & 2140 & 2160 \\
$\mathrm{DBO}_{5}\left(\mathrm{mgL}^{-1} \mathrm{~d}^{\prime} \mathrm{O}_{2}\right)$ & 1000 & 1500 & 750 & 800 & 500 & 500 \\
\hline
\end{tabular}

Tableau 2: Influence du débit de gaz plasmagène (à 10 min).

\begin{tabular}{|c|c|c|c|c|c|c|}
\hline Débit $\left(\mathbf{L h}^{-1}\right)$ & $\mathbf{0}$ & 200 & 400 & 600 & 800 & 1000 \\
\hline $\mathrm{T}\left({ }^{\circ} \mathrm{C}\right)$ & 28.2 & 31.7 & 30.7 & 31.1 & 31.2 & 30 \\
\hline $\mathrm{pH}$ & 8.65 & 9.04 & 9.16 & 9.18 & 9.19 & 9.21 \\
\hline Conductivité $\left(\mu \mathrm{Scm}^{-1}\right)$ & 1625 & 1906 & 1847 & 1782 & 1802 & 1716 \\
\hline $\operatorname{MES}\left(\mathrm{mgL}^{-1}\right)$ & 775 & 600 & 675 & 675 & 750 & 775 \\
\hline $\mathrm{DCO}\left(\mathrm{mgL}^{-1} \mathrm{~d}^{\prime} \mathrm{O}_{2}\right)$ & 2390 & 2090 & 2320 & 2490 & 2290 & 2340 \\
\hline $\mathrm{DBO}_{5}\left(\mathrm{mgL}^{-1} \mathrm{~d}^{\prime} \mathrm{O}_{2}\right)$ & 1000 & 2000 & 2100 & 2000 & 1800 & 500 \\
\hline
\end{tabular}

Tableau 3: Résultats d'autres paramètres analysés.

\begin{tabular}{lc}
\hline Paramètres & Teneur \\
\hline $\mathrm{Cl}^{-}\left(\mathrm{mgL}^{-1}\right)$ & 1347 \\
$\mathrm{SO}_{4}^{2-}\left(\mathrm{mgL}^{-1}\right)$ & $<5$ \\
$\mathrm{NO}_{2}^{-}\left(\mathrm{mgL}^{-1}\right)$ & $<0.02$ \\
$\mathrm{NH}_{4}^{+}\left(\mathrm{mgL}^{-1}\right)$ & 200 \\
$\mathrm{Na}^{+}\left(\mathrm{mgL}^{-1}\right)$ & 100 \\
$\mathrm{~K}^{+}\left(\mathrm{mgL}^{-1}\right)$ & 70 \\
$\mathrm{Ca}^{2+}\left(\mathrm{mgL}^{-1}\right)$ & 22 \\
$\mathrm{Mg}^{2+}\left(\mathrm{mgL}^{-1}\right)$ & 7
\end{tabular}




\begin{tabular}{lc}
$\left(\mathrm{Fe}^{2+}, \mathrm{Fe}^{3+}\right)\left(\mathrm{mgL}^{-1}\right)$ & $<0.02$ \\
$\mathrm{Mn}^{2+}\left(\mathrm{mgL}^{-1}\right)$ & $<0.02$ \\
$\mathrm{Cu}^{2+}\left(\mathrm{mgL}^{-1}\right)$ & $<0.02$ \\
$\mathrm{Zn}^{2+}\left(\mathrm{mgL}^{-1}\right)$ & $<0.02$ \\
$\mathrm{Ni}^{2+}\left(\mathrm{mgL}^{-1}\right)$ & $<0.1$ \\
$\mathrm{~Pb}^{2+}\left(\mathrm{mgL}^{-1}\right)$ & $<0.01$ \\
\hline
\end{tabular}

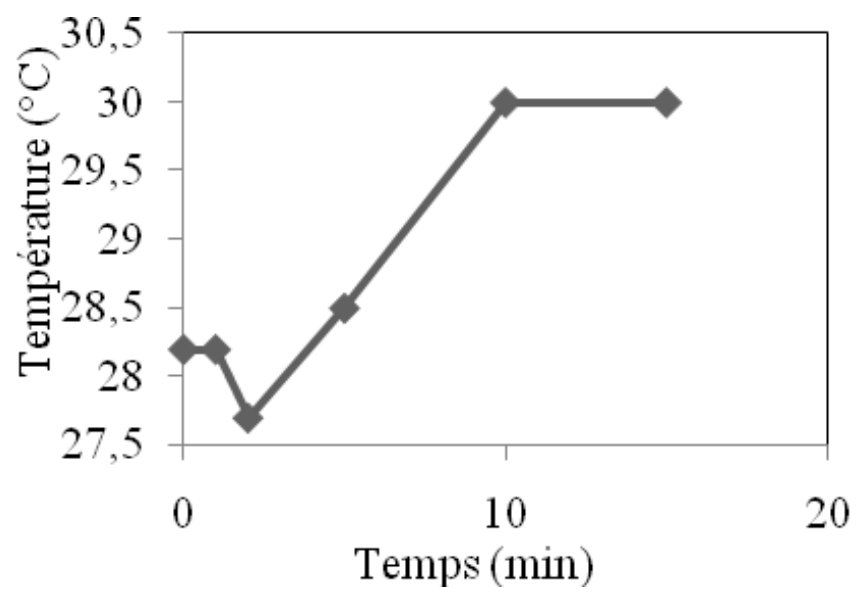

Figure 2: Evolution de la température en fonction du temps.

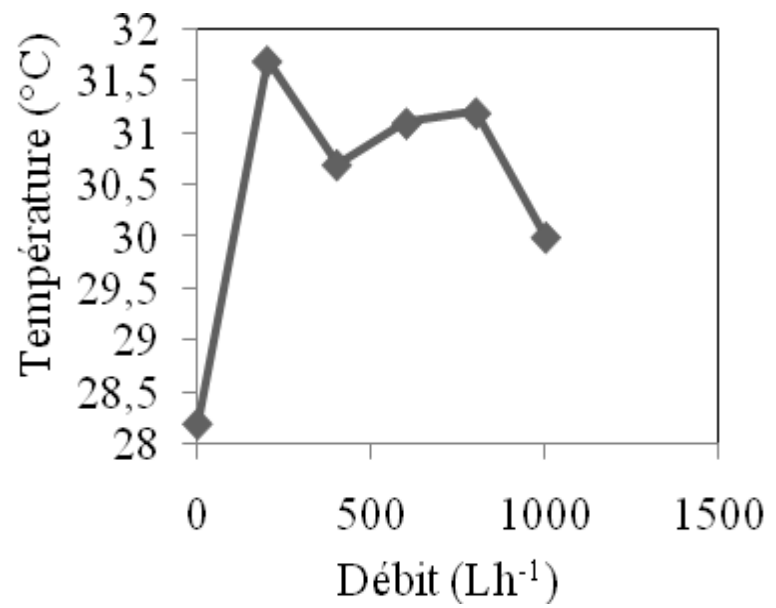

Figure 3: Evolution de la température en fonction du débit. 


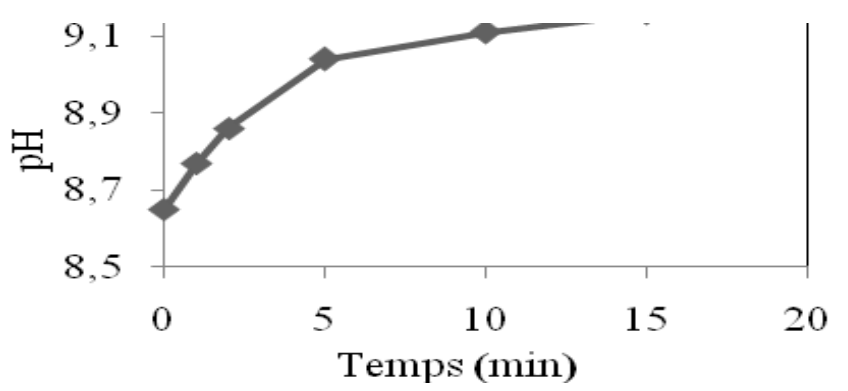

Figure 4 : Evolution du $\mathrm{pH}$ en fonction du temps.

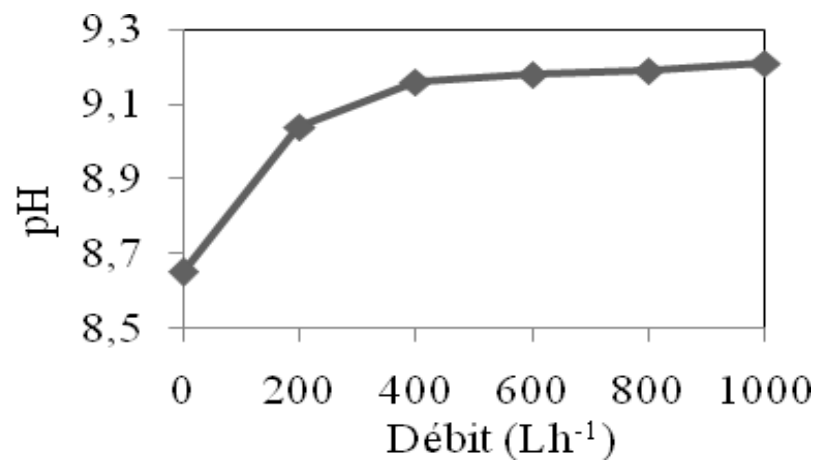

Figure 5 : Evolution du pH en fonction du débit.

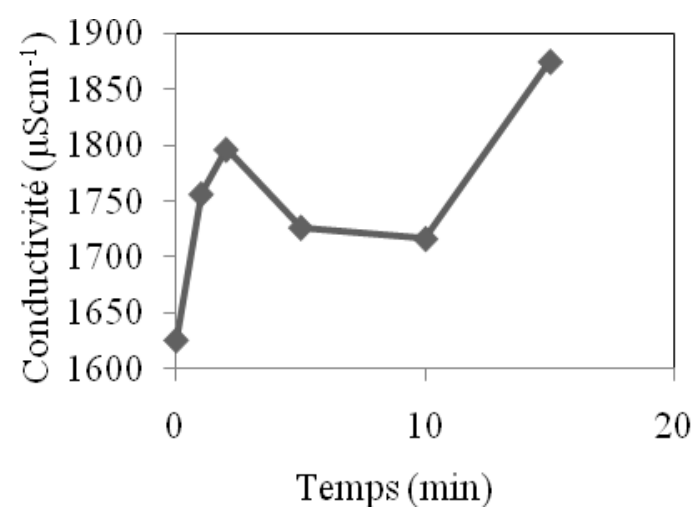

Figure 6 : Evolution de la conductivité en fonction du temps. 


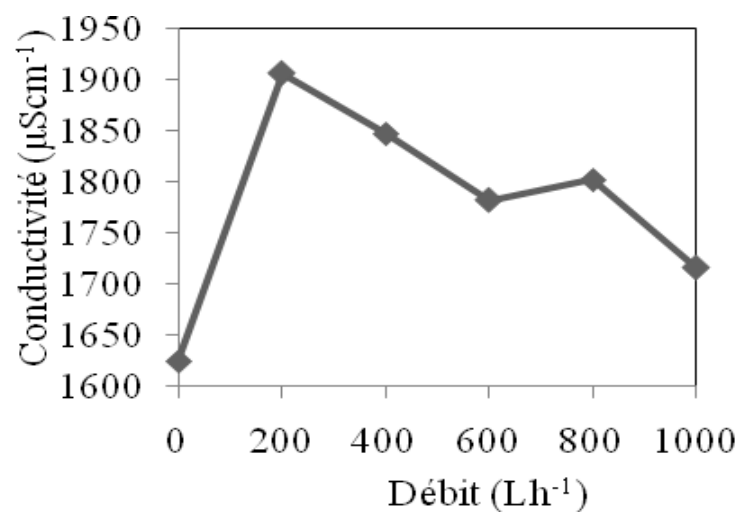

Figure 7 : Evolution de la conductivité en fonction du débit.

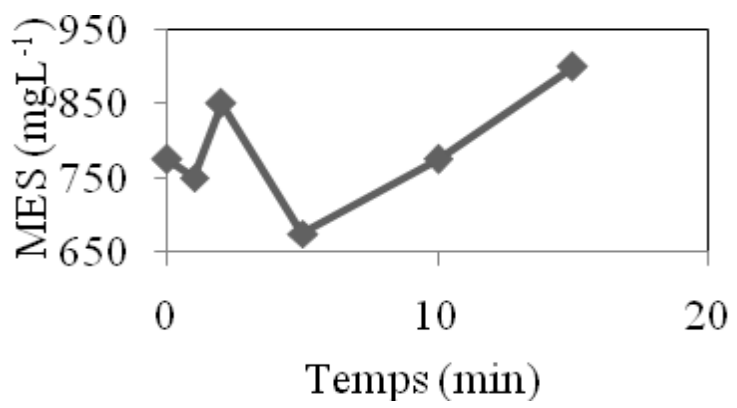

Figure 8: Evolution des MES en fonction du temps.

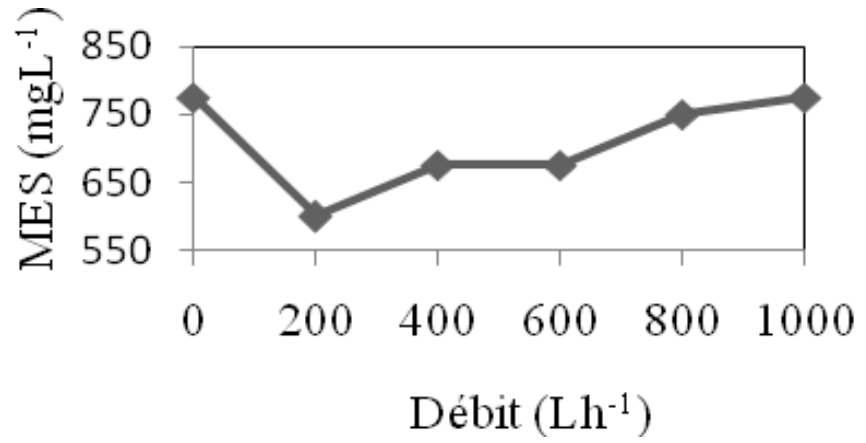

Figure 9 : Evolution des MES Figure en fonction débit. 


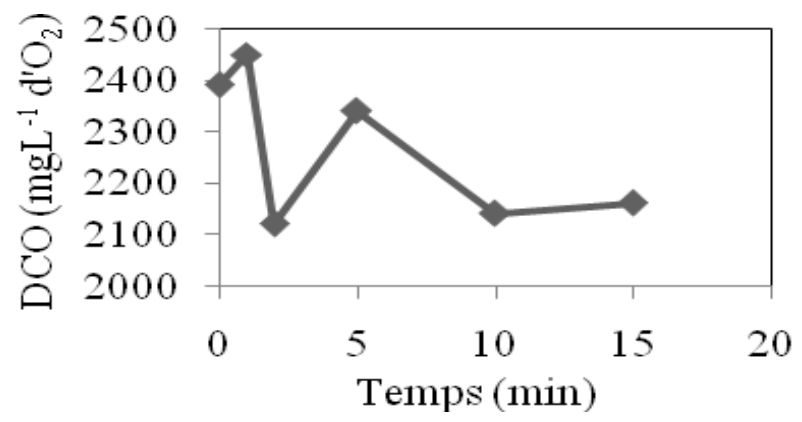

Figure 10: Evolution de la DCO en fonction du temps.

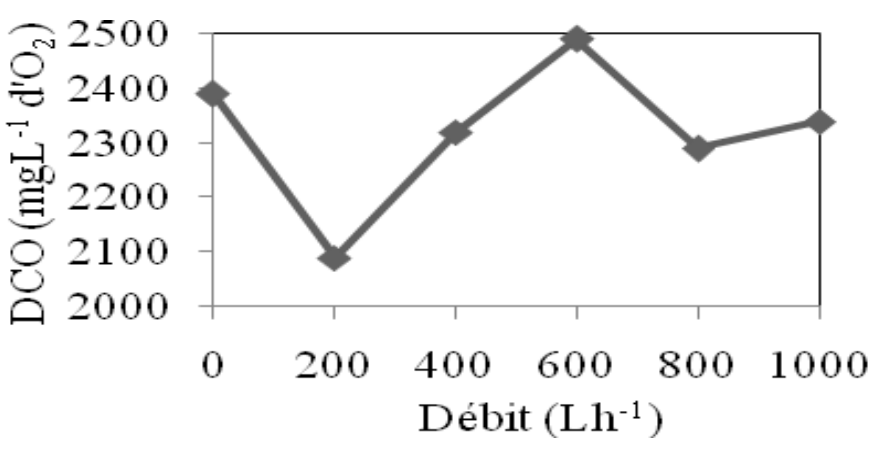

Figure 11 : Evolution de la DCO en fonction du débit.

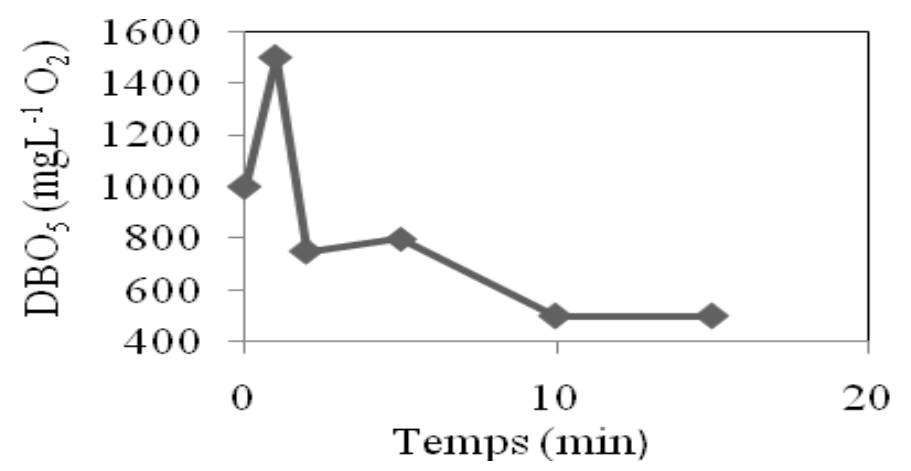

Figure 12: Evolution de la $\mathrm{DBO}_{5}$ en fonction du temps. 


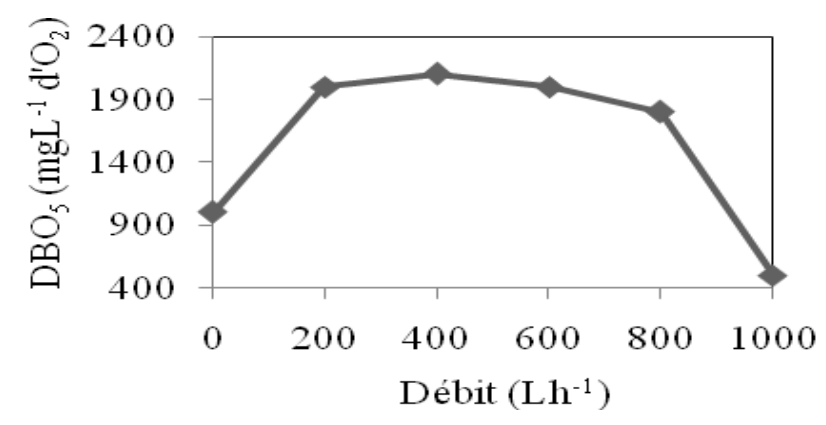

Figure 13 : Evolution de la $\mathrm{DBO}_{5}$ en fonction du débit.

\section{DISCUSSION}

La température croît avec le temps de traitement. Elle devient constante $\left(30{ }^{\circ} \mathrm{C}\right)$ à partir de 10 minutes. Cette évolution est conforme aux résultats antérieurs et à la norme française fixée à $30{ }^{\circ} \mathrm{C}$ (Rodier, 2009). Par ailleurs, la température reste relativement constante autour de $31{ }^{\circ} \mathrm{C}$ quelle que soit la variation du débit. Nous pouvons tirer comme conclusion que la température est davantage influencée par le temps de traitement que par la variation du débit en gaz plasmagène (Figures 2 et 3 ).

Que ce soit avec le temps de traitement ou avec le débit du gaz plasmagène, le pH de nos échantillons augmente curieusement alors que le plasma est reconnu comme ayant propriétés acidifiantes (Doubla et al., 2003 ; Moussa et al., 2005). Ce comportement du pH a été aussi observé par Gnokam Zumgang (2008) dans ses travaux de Thèse. En effet, l'existence des espèces hautement réactives comme le $\mathrm{NO}^{\circ}$ ou le $\mathrm{OH}^{\circ}$ ajouté à la composition des eaux de rejet des abattoirs pourraient être à l'origine de ces propriétés basiques. Le pH élevé des eaux usées de «l'abattoir des Brasseries» pourrait s'expliquer par la présence dans lesdites eaux des produits des abattoirs comme la graisse, la viande et surtout le sang dont le $\mathrm{pH}$ théorique est voisin de 8 (Gnokam Zumgang, 2008). Par ailleurs, l'évolution «anormale » du $\mathrm{pH}$ de nos échantillons présente un aspect très important qui mérite des investigations plus poussées. Cette évolution s'expliquerait par le fait que les temps de traitement et les débits en gaz plasmagène correspondent à une phase où les espèces basiques sont davantage produites dans le milieu, ce qui induit l'augmentation du $\mathrm{pH}$ (Figures 4 et 5). Cependant le $\mathrm{pH}$ garde des valeurs conformes à la norme française c'est-à-dire compris entre 5.5 et 9.5 (Rodier, 2009).

Les Figures 6 et 7 montrent que la conductivité électrique croît en dent de scie autant avec le temps qu'avec le débit atteignant des valeurs de $1906 \mu \mathrm{Scm}^{-1}$. Ceci pourrait s'expliquer par l'élévation de la température et ainsi se confirme la production des espèces de plus en plus chargées par le plasma (Doubla, 2002). Pour les eaux étudiées, la conductivité se trouve au-delà de la norme ghanéenne fixée à $1500 \mu \mathrm{Scm}^{-1}$ (Rodier, 2009).

$\mathrm{Si}$ nous pouvons voir que les MES croissent à partir de $200 \mathrm{Lh}^{-1}$ par rapport au débit, il n'est pas aisé d'expliquer leur variation en fonction du temps de traitement. On observe une augmentation particulièrement considérable à 15 minutes (900 $\mathrm{mgL}^{-1}$ ) par rapport à la valeur initiale (775 $\mathrm{mgL}^{-1}$ ). Dans tous les cas, les MES ont des teneurs hors norme (150 $\mathrm{mgL}^{-1}$ pour la France) d'après Rodier (2009). On pourrait s'attendre à une quantité appréciable des matières décantables pour que s'expliquent le niveau et la variation des MES (Figures 8 et 9).

La DCO qui traduit une pollution par toutes les matières oxydables en général, se 
trouve à un taux très appréciable dans les eaux de rejet de «l'abattoir des Brasseries» audelà de la norme Française $\left(300 \mathrm{mgL}^{-1} \mathrm{~d}^{\prime} \mathrm{O}_{2}\right)$ d'après Rodier (2009). Son évolution en fonction du temps de traitement comme en fonction du débit est difficile à expliquer (Figures 10 et 11). La présence des espèces comme les nitrites et les chlorures perturberait son évaluation. En effet, les électrons issus des réactions d'oxydoréduction de ces agents réducteurs coexistent et interfèrent avec ceux issus de l'oxygène normalement estimés lors du dosage (Rodier, 2009). On note tout de même une substantielle réduction de la DCO à partir de $10 \mathrm{mn}$ de traitement (Figure 10).

La $\mathrm{DBO}_{5}$, qui est une évaluation de la pollution organique biodégradable est très élevée dans ces eaux ; elle est au-delà de la norme Française $\left(100 \mathrm{mgL}^{-1} \mathrm{~d}^{\prime} \mathrm{O}_{2}\right)$ d'après Rodier (2009). Cependant, en 10 minutes de traitement nous observons une réduction de $50 \%$ de la $\mathrm{DBO}_{5}$ (Figure 12). Sa teneur décroît avec le temps de traitement après une augmentation brusque entre 0 et 1 minute passant de 1000 à $1500 \mathrm{mgL}^{-1} \mathrm{~d}^{\prime} \mathrm{O}_{2}$. Par contre, avec un débit de gaz de 200 à $800 \mathrm{Lh}^{-1}$, la $\mathrm{DBO}_{5}$ reste pratiquement constante avant de chuter à $1000 \mathrm{Lh}^{-1}$ (Figure 13). L'influence du temps est plus significative que celle du débit. Par ailleurs, le rapport $\mathrm{DBO}_{5} / \mathrm{DCO}$ est toujours inférieur à l'unité, alors les eaux usées de «l'abattoir des Brasseries » sont biodégradables (Rodier, 2009). On peut donc affirmer que le plasma froid à décharge électrique rampante réduit efficacement la $\mathrm{DBO}_{5}$.

Des paramètres complémentaires ont été analysés à partir de l'échantillon brut mais non suivis au cours des traitements pour des raisons d'ordre matériel. Ce sont notamment les chlorures, les sulfates, les nitrites et les métaux (Tableau 3). Il ressort de ce tableau que les chlorures ont un taux élevé (soit 1347 $\mathrm{mgL}^{-1}$ ) comparée à la norme Ghanéenne limitée à $250 \mathrm{mgL}^{-1}$ (Rodier, 2009). La présence des chlorures qui sont d'origine atmosphérique est liée à celle des ions potassium, calcium, sodium, magnésium et ammonium présents et avec lesquels on peut avoir les paires $\left(\mathrm{K}^{+}, \mathrm{Cl}^{-}\right)$; $\left(\mathrm{Na}^{+}, \mathrm{Cl}^{-}\right)$; $\left(\mathrm{Ca}^{2}\right.$, $\left.2 \mathrm{Cl}^{-}\right)$; $\left(\mathrm{Mg}^{2+}, 2 \mathrm{Cl}^{-}\right)$et $\left(\mathrm{NH}_{4}{ }^{+}, \mathrm{Cl}^{-}\right)$. Les ions sodium, potassium, calcium et magnésium en quantité notable peuvent avoir une origine géologique donc contenus dans l'eau utilisée pour le lavage des produits consommables de l'abattoir. Les ions ammonium par contre traduisent une pollution d'origine agricole et proviendraient du contenu des viscères. Il est à noter l'absence des métaux lourds dans les eaux de rejet de «l'abattoir des Brasseries ». Cette absence renforcerait les allégations précédentes concernant la prépondérance de la pollution organique desdites eaux. On peut, par ailleurs, comprendre à partir de la concentration élevée des chlorures, la difficulté à pouvoir mesurer normalement la $\mathrm{DCO}$ et de ce fait son évolution, à cause des interférences électroniques (Rodier, 2009).

\section{Conclusion}

Des résultats d'analyse de l'échantillon brut, il ressort que les eaux usées de « l'abattoir des Brasseries » sont basiques $(\mathrm{pH}$ $=8.65)$, très conductrices $\left(1625 \mu \mathrm{Scm}^{-1}\right)$, fortement polluées $\left(\mathrm{DBO}_{5}=1000 \mathrm{mgL}^{-1} \mathrm{~d}^{\prime} \mathrm{O}_{2}\right.$, $\mathrm{DCO}=2390 \mathrm{mgL}^{-1} \mathrm{~d}^{\prime} \mathrm{O}_{2}$ et $200 \mathrm{mgL}^{-1}$ d'ammonium) et contiennent des substances biodégradables mais pas de métaux lourds.

Le traitement par plasma froid à décharge électrique rampante (glidarc) a donné des résultats encourageants en ce qui concerne la réduction de la $\mathrm{DBO}_{5}$ et de la DCO. Par ailleurs, les résultats obtenus nous amènent à comprendre que la fixation par exemple du débit en gaz plasmagène à 1000 $\mathrm{Lh}^{-1}$ permet d'obtenir un très bon rendement en temps réduit. Ces résultats prouvent une fois de plus l'efficacité du plasma froid de type glidarc à réduire considérablement la pollution organique des eaux des abattoirs. Cependant, le comportement basique du $\mathrm{pH}$ au cours du temps de traitement ouvre des voies à plus d'investigations.

\section{REMERCIEMENTS}

Ce travail a été réalisé grâce au laboratoire de Chimie Minérale et au laboratoire de Phyto-épuration, tous deux à 
l'Université de Yaoundé I (Cameroun) sans oublier le laboratoire du Ministère de l'Eau et de l'Energie du Cameroun. A tous les responsables de ces structures nous adressons nos sincères remerciements.

\section{REFERENCES}

Brisset JL. 2009. La foudre en bouteille. Décharges électriques glissantes à la pression atmosphérique et leurs applications à l'environnement. Le Bup, 912(103): 257-280.

Doubla A, Abdelmalek F, Khélifa K, Addou A, Brisset JL. 2003. Post-discharge plasma-chemical oxidation of Iron(II) complexes. Journal of Applied Electrochemistry, 33: 73-77.

Doubla A. 2002. Propriétés oxydo-réductrices des plasmas non thermiques d'air humide : application à la dépollution des eaux et à la corrosion métallique. Diplôme d'Habilitation à Diriger les Recherches, Université de Rouen.

Fridman A. 2008. Plasma Chemistry. Cambridge University Press: New York.
Gnokam Zumgang FDS. 2008. Dégradation de l'hémoglobine et des effluents d'abattoirs par décharge électrique glissante dans l'air humide à pression atmosphérique. Thèse de Doctorat en cotutelle, Université de Rouen-Université de Yaoundé I, Rouen.

Held B. 1994. Physique des Plasmas Froids. Edn. Masson: Paris.

Labioui H, Elmoualdi L, Benabbou Y, Elyachioui M, Ouhssine M. 2007. Traitement et valorisation de déchets en provenance d'abattoirs au Maroc. Agrosolutions, 18(1): 35-40.

Moussa D, Abdelmalek F, Benstaali B, Addou A, Hnatiuc E, Brisset JL. 2005. Acidity control of the gliding arc treatments of aqueous solutions: application to pollutant abatement and biodecontamination. Eur. Phys. J. Appl. Phys., 29: 189-199.

Rodier J. 2009. L'Analyse de l'Eau (9 $9^{\mathrm{e}}$ edn). Dunod: Paris. 